\title{
Estratégia de utilização da folga de tração de trens para planejamento da distribuição de vagões vazios
}

\section{Patricia Costa Louzada1, Rodrigo de Alvarenga Rosa², Matheus Batista Foletto ${ }^{3}$, Lucas de Barros Pimenta ${ }^{4}$}

1Universidade Federal do Espírito Santo, Programa de Pós-Graduação em Engenharia Civil, patylouzada@hotmail.com 2Universidade Federal do Espírito Santo, Programa de Pós-Graduação em Engenharia Civil, rodrigoalvarengarosa@gmail.com 3Universidade Federal do Espírito Santo, Departamento de Engenharia de Produção, matfoletto@gmail.com

${ }^{4}$ Universidade Federal do Espírito Santo, Programa de Pós-Graduação em Engenharia Civil, lucas.barrospimenta@gmail.com

\section{Recebido:}

4 de dezembro de 2017

Aceito para publicação:

15 de maio de 2018

Publicado:

31 de agosto de 2018

Editor de área:

Marcio D'Agosto

\section{Palavras-chaves:}

Distribuição de vagões vazios,

Operação ferroviária,

Transporte ferroviário.

\section{Keywords:}

Distribution of empty wagons,

Railway transport,

Railway transport.

DOI:10.14295/transportes.v26i2.1537

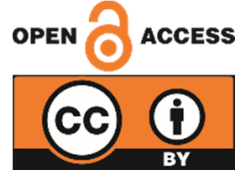

\begin{abstract}
RESUMO
No transporte ferroviário, o planejamento da distribuição de vagões vazios é importante porque a maioria dos vagões nas ferrovias brasileiras retornam vazios do pátio de descarga para o pátio de carregamento não gerando receita para a ferrovia e gerando mais custos com maquinista e combustível. Assim, este artigo propõe um modelo matemático de Programação Inteira que se baseia numa estratégia para distribuição de vagões vazios, onde esses são transportados utilizando a folga na capacidade de tração dos trens que circularão com vagões carregados já programados para atendimento às demandas dos pátios. Para avaliar o modelo proposto, foram criadas instâncias baseadas em dados reais da Ferrovia Centro-Atlântica (FCA) que foram resolvidas de forma ótima utilizando o solver CPLEX 12.6. O modelo matemático utilizando a estratégia proposta apresentou redução na formação de trens exclusivos de vagões vazios quando comparada com o planejamento manual da ferrovia.
\end{abstract}

\section{ABSTRACT}

Planning the distribution of empty wagons in rail transport is important because most wagons on Brazilian railways return empty from the unloading rail yard to the loading rail yard without bringing any revenue for the railway and adding costs due to engine driver and diesel. Therefore, this article proposes an Integer Programming mathematical model based on a strategy for the distribution of empty wagons, where they are distributed using the non-used traction capacity of the trains which will travel with loaded wagons. To evaluate the proposed model, instances were created based on real data from Central Atlantic Railroad (FCA). They were optimally solved using CPLEX 12.6 solver. The mathematical model using the proposed strategy presented reduction in making up trains only with empty wagons compared to the manual planning of the railway.

\section{INTRODUÇÃO}

0 transporte ferroviário de cargas cresceu 32,7\% entre os anos de 2007 e 2016 medido em tonelada por quilômetro útil (TKU), chegando à marca de 341,2 bilhões de TKU em uma extensão total de $30.576 \mathrm{~km}$ (CNT, 2017).

0 transporte ferroviário no Brasil se caracteriza por carregar os vagões em um pátio de origem (mina, armazém agrícola, entre outros), realizar o transporte até um pátio de destino, descarregar em um pátio de descarga (um porto, um centro de distribuição) e retornar vazio para o pátio de origem para carregar. Esse retorno com o vagão vazio eleva os custos operacionais da ferrovia, pois um trem exclusivamente formado de vagões vazios acarreta custos com maquinista e combustível, além de não auferir receita para a ferrovia. 
Visando reduzir o número de trens circulando somente com vagões vazios, esse artigo tem como objetivo propor um modelo matemático de Programação Inteira para planejamento da distribuição de vagões vazios entre pátios ferroviários para atendimento à demanda de vagões vazios para carregamento utilizando a estratégia de aproveitar a folga na capacidade de tração dos trens carregados visando a minimização do custo de distribuição. Essa estratégia de distribuir vagões vazios pode levar à redução da formação de trens apenas com vagões vazios. 0 modelo matemático define a quantidade e tipo de vagões vazios a serem transportados entre pátios com oferta e com demanda e determina em quais trens que já iriam circular transportando vagões carregados serão alocados tais vagões vazios.

Para avaliar o modelo matemático proposto, o modelo foi testado com dados da Ferrovia Centro-Atlântica (FCA). Os resultados alcançados pelo CPLEX foram comparados com a distribuição de vagões vazios realizada pelos responsáveis pela distribuição de vagões vazios da FCA no período em que foi analisado. Os resultados mostram que a solução alcançada pelo CPLEX rodando o modelo matemático proposto reduziu o número de trens formados exclusivamente de vazios caso a solução encontrada fosse aplicada na prática.

Além da presente seção, de caráter introdutório, este artigo é constituído por outras cinco seções. Na Seção 2, é apresentado o referencial teórico sobre os principais conceitos relativos ao Problema de Distribuição de Vagões Vazios. A Seção 3 apresenta o modelo matemático proposto. 0 levantamento de dados e as instâncias de testes são apresentadas na Seção 4. Na Seção 5, são apresentados os resultados e as análises. Por fim, na Seção 6 são apresentadas as conclusões do artigo.

\section{O PROBLEMA DA DISTRIBUIÇÃO DE VAGÕES VAZIOS}

O problema da distribuição de vagões vazios consiste em transportar vagões vazios de pátios onde existe oferta para pátios onde existe demanda. Para isso, são definidas as origens e destinos dos vagões vazios para atender a demanda de transporte e, em seguida, são definidos em quais trens que circularão na ferrovia serão transportados os vagões vazios entre as origens e destinos conhecidos.

A solução leva em consideração a quantidade e a localização inicial dos vagões vazios, ou seja, em quais pátios e tempos existem ofertas de vagões vazios, e a quantidade e tipos de vagões vazios solicitados para carregamento, ou seja, em quais pátios e tempos existem demandas de vagões vazios. 0 problema da distribuição de vagões vazios visa atender a demanda de transporte objetivando a minimização dos custos de distribuição de vagões.

A abordagem mais tradicional para tratar o problema de distribuição de vagões vazios é a utilização de uma rede espaço-tempo para representar a operação de trens, entre pátios, em certo horizonte de tempo (CRAINIC e LAPORTE, 1997). Na rede espaço-tempo, o horizonte de planejamento é dividido em tempos discretizados que podem ser um dia ou um turno de trabalho ou mesmo uma hora. 0 fluxo nessa rede representa o movimento de vagões vazios de um pátio para outro ou vagões vazios estacionados em um mesmo pátio ao longo do tempo (JOBORN et al., 2004), como representado na Figura 1. Cada nó na rede espaço-tempo representa um pátio em um certo momento de tempo e pode ocorrer neste nó uma oferta de ou uma demanda por vagões vazios.

A Figura 1 ilustra o fluxo de vagões entre os pátios em uma ferrovia. 0 segmento entre o (pátio 1, tempo 1) e o (pátio 2, tempo 2) representa um trem iniciando sua circulação no pátio 
1 no tempo 1 com destino ao pátio 2 no tempo 2 transportando uma quantidade de vagões vazios. Entre os tempos 2 e 3, a representação significa que uma certa quantidade de vagões ficou estacionado no pátio 2 nesse período. 0 segmento final representa um trem iniciando sua circulação no pátio 2 no tempo 3 com destino ao pátio 3 no tempo 4, transportando uma certa quantidade de vagões esse trecho.

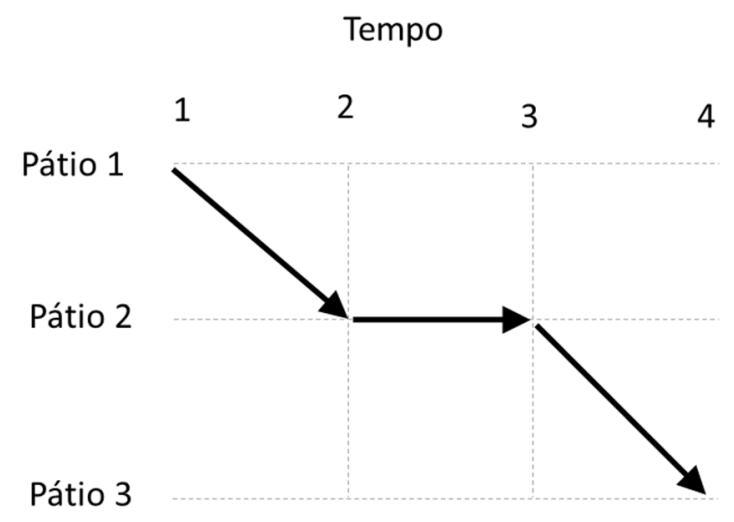

Figura 1. Rede Espaço-Tempo

Na representação da rede espaço-tempo, o número de colunas é igual ao horizonte de tempo discretizado considerado no problema e o número de linhas é igual à quantidade de pátios da ferrovia considerada. Os nós representam os pátios em um tempo específico. Os trens saem de um pátio de origem em certo tempo, para um pátio de destino em outro tempo subsequente. Desse modo, a rede espaço-tempo representa os movimentos dos trens na forma de uma sequência origem-destino, por meio de um par ordenado, indicando respectivamente: pátio de origem e tempo de origem, e pátio de destino e tempo de destino.

Dentro do contexto da distribuição de vagões vazios, vários artigos foram publicados na literatura. Um dos primeiros artigos na área de gestão dos vagões vazios foi desenvolvido por White e Bomberault (1969), que considera o problema da distribuição de vagões vazios em toda a rede, dada a previsão de demanda. Um modelo de programação linear de fluxo de rede é proposto para reposicionar vagões, a um custo mínimo, para os locais onde eles são necessários para atender a demanda. Misra (1972) estudou a distribuição estática e considerou a questão como um problema de transporte, porém não considerou o fator tempo e assumiu o fornecimento da demanda equilibrada em cada dia. Como a distribuição dependia do fator tempo, e este foi ignorado, a teoria estática foi pouco estudada.

Philip (1978) utilizou modelos de simulação para distribuir os vagões vazios e analisar as operações de pátio. Dejax e Crainic (1987) reuniram e revisaram em um único artigo os modelos de gerenciamento e fluxo da frota de vagões vazios. Eles consideraram o deslocamento de vagões vazios como um problema exclusivo de custo, pois nessa situação os vagões não geram receita e é por essa razão que o planejamento da distribuição de vagões consiste na redução da circulação desses ativos na condição de vazio.

Uma formulação e solução que combina roteamento, formação de trens e alocação de vagões vazios foi proposta por Haghani (1989). Tal formulação resultou em um modelo de programação inteira mista, com uma função objetivo não linear, e restrições lineares. Para resolução do modelo foi desenvolvida uma técnica de decomposição heurística. 
Spieckermann e Voß (1995) afirmaram que os modelos matemáticos e estudos teóricos estão disseminados nas literaturas específicas, mas que ainda necessitam de serem aplicados no mundo real. Eles desenvolveram uma heurística de distribuição de vagões para uma empresa alemã de aluguel de vagões. Cordeau, Toth e Vigo (1998) fizeram uma revisão da maioria dos modelos propostos durante a década de 80 , onde tratam da distribuição de vagões vazios, rotas e programação de trens (tanto de carga, como de passageiro). 0 estudo fez uma abordagem aos três níveis de planejamento, sendo a atenção concentrada em problemas globais de gerenciamento dos trens. Powell e Carvalho (1998) estudaram o problema da gestão de frota de vagões numa ferrovia em um ambiente de tempo real.

Crainic e Laporte (1997) apresentaram importantes questões nos sistemas de planejamento e gerenciamento no transporte de carga, abordando todos os três níveis de planejamento: estratégico, tático e operacional. Em cada nível foi feita uma revisão bibliográfica, descrevendo os principais problemas relacionados e como as questões foram abordadas por meio do desenvolvimento em modelos de pesquisa operacional. No nível operacional, eles destacam a modelagem matemática em uma rede espaço-tempo, na qual representa os trens, os pátios, o horizonte de tempo de planejamento, e por fim, o fluxo de vagões vazios.

Fukasawa (2002) abordou os problemas de nível tático e operacional. No problema tático, foi abordado o Problema de Planejamento de Atendimento (PPA), onde o objetivo foi definir as diretrizes de atendimento que serviriam como base para todo o planejamento da operação mensal. Neste nível tático, levou-se em conta: a capacidade da malha, o tamanho da frota de vagões, blocagem, classes de vagões, disponibilidade de vagões vazios, entre outros aspectos. Para este problema, foi desenvolvido um modelo mono-periódico baseado no modelo de multifluxos. No nível operacional, foi analisado o chamado Problema de Fluxo de Vagões (PFV), no qual foi determinada a rota completa de cada vagão na malha ferroviária, bem como a sequência de carregamentos e descarregamentos, no intervalo de uma semana. Neste modelo, o objetivo foi definir quando e como atender, ou não, cada demanda, de modo a maximizar o lucro total. Para o PFV, foi proposto um modelo baseado em multi-fluxos, porém dessa vez periódico, sendo que nele são contemplados todos os possíveis movimentos e operações de vagões que podem ser feitos no período. Para ambos os modelos foi usado o solver CPLEX 7.1.

Segundo Joborn et al. (2004), o problema de distribuição de vagões vazios inclui os problemas de planejamento e desempenho da movimentação de vagões vazios, objetivando a minimização dos custos, de modo a satisfazer a oferta e a demanda existentes. Os autores ainda afirmaram que se menos, porém maiores, grupos de vagões pudessem ser atendidos nos pátios, não apenas os processos de manobra e classificação seriam simplificados, mas também o custo unitário do vagão associado seria diminuído, uma vez que a estrutura de custo exibe um comportamento de economia de escala.

Uma introdução à gestão de frotas com foco na distribuição de vagões pode ser encontrada em estudos de Powell e Topaloglu (2005), Powell et al. (2007) e Holmberg, Joborn e Lundgren (1998), os quais propuseram modelos de otimização para melhorar o processo de distribuição de vagões vazios para atender o agendamento de minas de minério de ferro. Bektas, Cranic e Morency (2009) tiveram por objetivo reduzir o tempo em que os vagões vazios ficam parados nos pátios. Quanto à Sherali e Suharko (1998), estes discutiram estratégias de reposicionamento dos vagões vazios para transporte de automóveis.

Melo, Barros e Nobre (2008) estudaram o problema de planejamento da alocação de vagões de carga por meio de um modelo de Programação Inteira Mista. 0 horizonte de planejamento 
estudado foi de quinze dias, sob o ponto de vista do plano operacional, ou seja, a curto prazo. Em seu artigo eles descreveram cinco modelos, nos quais as funções objetivo solucionavam cinco problemas distintos: minimização dos vagões ociosos retidos em cada pátio, minimização do número de vagões em circulação (vazios e carregados), minimização dos vagões em circulação (apenas vazios), maximização do lucro e a minimização dos custos.

Costa (2010) abordou o problema de distribuição e alocação de vagões vazios em duas etapas distintas. Primeiramente, foi realizado o processo de distribuição de vagões, definindo os destinos de cada vagão para atender a demanda de transporte. Em seguida foi gerada uma solicitação de movimentação de vagões, a qual serve de dado de entrada para a segunda parte do problema, que foi definir quais trens transportarão os vagões de sua origem até o seu destino (alocação dos vagões). Para este último problema, foi elaborado um algoritmo de alocação, cujo objetivo foi encontrar algum trem, dentro da grade de trens, para levar os vagões da origem ao destino.

Após essa revisão da literatura, não foram encontrados artigos que propusessem utilizar a folga de tração dos trens formados de vagões carregados para transportar vagões vazios como é proposto neste artigo.

\section{MODELO MATEMÁTICO PROPOSTO}

Nessa seção, será descrito o modelo matemático proposto baseado numa rede espaço-tempo para distribuição de vagões vazios em uma ferrovia. Propõe-se neste artigo uma adaptação dos modelos de rede espaço-tempo propostos por Crainic e Laporte (1997) e Joborn et al. (2004). Na rede espaço-tempo proposta por estes autores, cada nó é representado por um par ordenado (pátio, tempo) (Figura 2a). Neste artigo, é considerado para cada nó um índice único, criando um vetor espaço-tempo, visando simplificar a estrutura de dados do problema (Figura $2 \mathrm{~b}$ ).

Para realizar a transformação da matriz espaço-tempo em vetor espaço-tempo, define-se $n p$ como o número de pátios da ferrovia e $p$ um pátio específico da ferrovia, sendo que $p$ varia de 0 a $n p-1$, ht o horizonte de planejamento e $t$ um tempo específico e discretizado variando de 1 até $h t$. Têm-se então duas situações: 1) quando se está no pátio $p$ no tempo $t=1$; e 2) quando se está no pátio $p$ no tempo $t>1$. Tomando as duas situações apresentadas anteriormente, pode-se transformar a rede espaço-tempo da Figura 2a no vetor espaço-tempo da Figura $2 \mathrm{~b}$ por meio da Equação (1).

$$
\omega=t+(p h t)
$$

Na Figura 2 é apresentado um exemplo com cinco pátios e um horizonte de planejamento de cinco dias e são apresentados dois exemplos, um para cada uma das duas situações apresentadas. Primeiro, analisando o pátio 0 no tempo 1 da Figura 2a chega-se à posição 1 do vetor espaço-tempo da Figura 2b calculada pela fórmula $\omega=t+(p h t)=1+(0 \cdot 5)=1$. Segundo exemplo, analisando o pátio 2 no tempo 3 da Figura 2 a chega-se à posição 13 do vetor espaçotempo da Figura 2b calculada pela fórmula $\omega=t+(p h t)=3+(2 \cdot 5)=13$. 0 valor de $\omega$ é limitado pelos valores $1 \leq \omega \leq(n p h t)$. Assim, após toda a transformação, conforme exemplificado anteriormente, chega-se, então, à Figura $2 \mathrm{~b}$ que representa o vetor espaço-tempo do problema da Figura 2a.

Nesse artigo os arcos representam o trem carregado e sua folga de capacidade de tração entre os pátios $i$ (nó de partida) e $j$ (nó de chegada) ou trens exclusivos de vagões vazios introduzidos pelos planejadores com custo muito superior a um trem carregado que vai somente acoplar vagões vazios na sua cauda. Como premissas da rede espaço-tempo, têm-se que: 1) um trem não pode realizar uma viagem entre pátios em um mesmo período de tempo (Figura 3a), 
ou para períodos de tempo anteriores (Figura 3b); 2) para os casos em que deve ser restringida a possibilidade de circulação de trens, o parâmetro folga de capacidade para estes arcos deve ser igual a zero, de modo que, onde houver folga de capacidade de tração entende-se que há algum trem planejado para o trajeto correspondente; 3) em cada arco só pode trafegar um trem, correspondente a uma seção de bloqueio na ferrovia; 4) não existem viagens dentro de um mesmo pátio, neste caso o trem manteve-se estacionado nas linhas de pátio (Figura 3c).

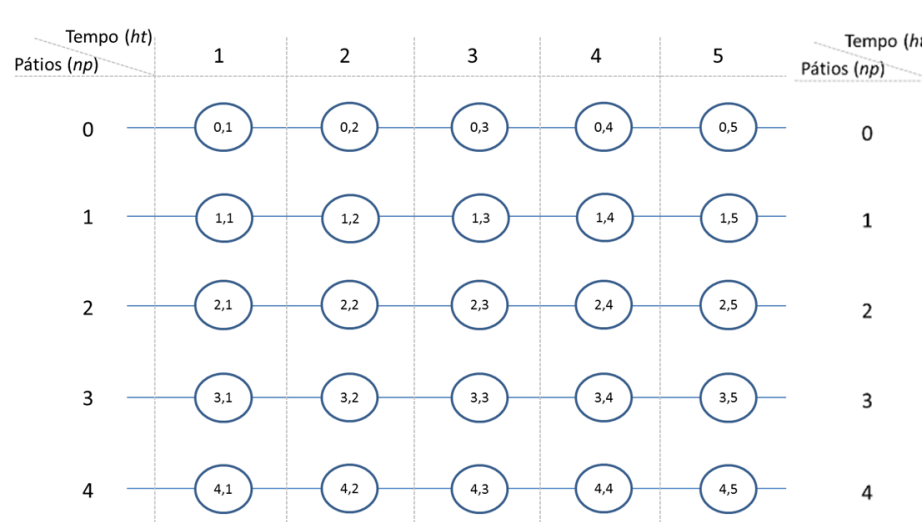

(a)

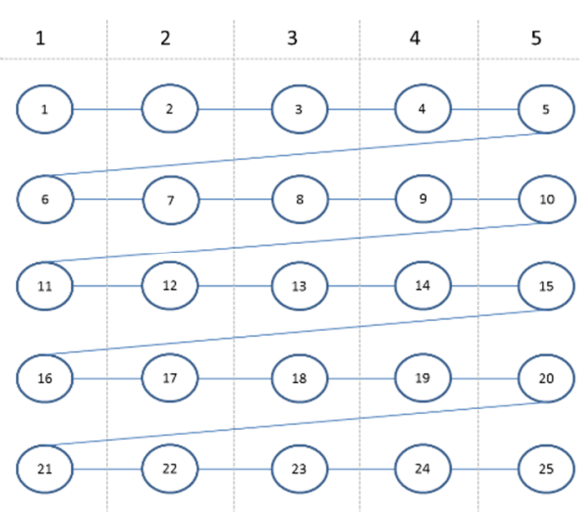

(b)

Figura 2. Representação da Rede Espaço-Tempo. a) par ordenado apresentado por Crainic e Laporte (1997) e Joborn et al. (2004) e b) vetor Espaço-tempo utilizado no modelo matemático proposto.

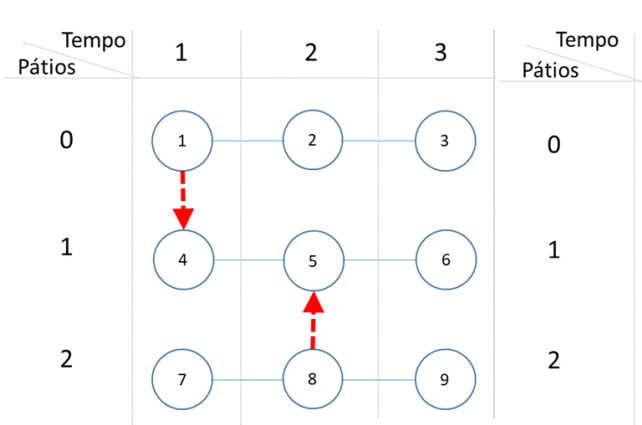

(a)

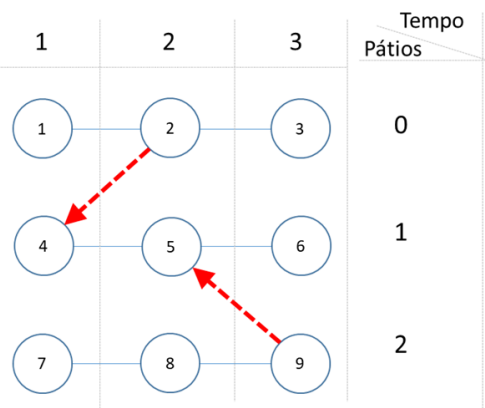

(b)

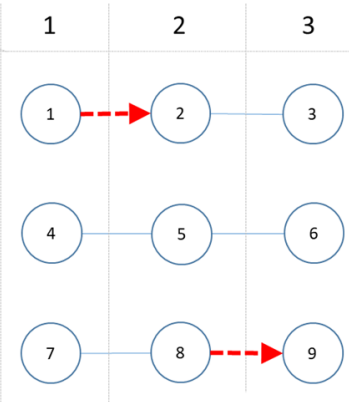

(c)

Figura 3. Representação dos arcos que assumem valor zero. a) viagens entre pátios no mesmo período de tempo, b) viagens para períodos de tempo anteriores e c) viagens dentro de um mesmo pátio

No modelo proposto, são previstos alguns trens exclusivos para distribuição de vagões vazios, sendo atribuído a estes trens, custos de circulação muito elevados. Essa estratégia foi adotada pois a folga de capacidade de tração dos trens circulando pode ser menor que a demanda para distribuir vagões vazios e, portanto, existem situações em que obrigatoriamente deverão existir trens exclusivamente de vazios.

0 modelo matemático está baseado em um vetor espaço-tempo, conforme Figura $2 \mathrm{~b}$. Este vetor espaço-tempo é constituído de $n p$ pátios e horizonte de planejamento de $h t$ tempos. 0 conjunto de nós do vetor espaço-tempo, portanto, é definido por $N=(1, \ldots,(n p h t))$. São considerados $v$ tipos de vagões disponíveis. A seguir, o modelo matemático proposto é apresentado em cinco partes: os conjuntos, os parâmetros, as variáveis de decisão, a função objetivo e as restrições.

\section{Conjuntos}

$P$ - Conjunto de pátios da ferrovia, variando de $0 \cdots(n p-1)$; 
$T$ - Conjunto de tempos discretizados, variando de $1 \cdots h t$;

$N$ - Conjunto de nós do vetor espaço-tempo, variando de $1 \cdots(n p h t)$;

$V$ - Conjunto de tipos de vagões, variando de $1 \cdots v$.

\section{Parâmetros}

$\sigma_{i j} \sigma g i j$ - Custo de transportar um vagão vazio no trecho $i \in N \mathrm{i} \in \mathrm{Na} j \in N \mathrm{j} \in \mathrm{N}$;

$\tau_{v} \tau \vartheta$ - Peso do tipo de vagão $v \in V \vartheta \in \mathrm{V}$;

$\varphi_{i j} \varphi$ gij - Folga de tração disponível do trem no trecho $i \in N \mathrm{i} \in \mathrm{Na} j \in N \mathrm{j} \in \mathrm{N}$;

$\theta_{v i} \theta \vartheta \mathrm{i}$ - Oferta de vagões vazios do tipo $v \in V$ no nó $i \in N$;

$\alpha_{v i} \alpha \vartheta \mathrm{i}$ - Demanda de vagões vazios do tipo $v \in V$ no nó $i \in N$;

$v_{v}$ - Limite máximo de vagões em um trem no trecho $i \in N \mathrm{i} \in \mathrm{Na} j \in N \mathrm{j} \in \mathrm{N}$;

$\rho_{i j}$ - Quantidade atual de vagões no trem percorrendo o trecho $i \in N \mathrm{i} \in \mathrm{Na} j \in N$.

\section{Variáveis de decisão}

$\gamma_{v i j} \gamma g \vartheta \mathrm{ij}$ - Quantidade de vagões vazios do tipo $v \in V$ que serão transportados acoplados em um trem no trecho $i \in N \mathrm{i} \in \mathrm{Na} j \in N$;

$\beta_{v i} \beta \vartheta \mathrm{i}$ - Número de vagões vazios do tipo $v \in V$ estacionados no nó $i \in N$.

\section{Função Objetivo}

Minimizar

$\sum_{i \in N} \sum_{j \in N} \sigma_{i j} \sum_{v \in V} \gamma_{v i j}$

Sujeito a:

$$
\begin{array}{ll}
\sum_{v \in V} \gamma_{v i j} \tau_{v} \leq \varphi_{i j} & \forall i, j \in N: i \neq j \\
\beta_{v(1+(p h t))}=\theta_{v(1+(p h t))}-\sum_{j \in N} \gamma_{v(1+(p h t)) j} & \forall v \in V, p \in P \\
\beta_{v(t+(p h t))}=\beta_{v((t+(p h t))-1)}+\theta_{v(t+(p h t))} & \\
\quad-\sum_{j \in N} \gamma_{v(t+(p h t)) j}+\sum_{j \in N} \gamma_{v j(t+(p h t))}-\alpha_{v(t+(p h t))} & t \in T: t>1 \\
\gamma_{v i j}+\rho_{i j} \leq \mu_{i j} & \\
\gamma_{v i i}=0 & \forall v \in V, i, j \in N \\
\beta_{v i} \in \mathbb{Z}^{+} & : i \neq j \\
\gamma_{v i j} \in \mathbb{Z}^{+} & \forall v \in V, i \in N \\
& \forall v \in V, i \in N \\
& \forall v \in V, i, j \in N
\end{array}
$$

A função objetivo (2) expressa o custo total de distribuição de vagões vazios, multiplicando o custo de distribuição de um vagão vazio $v \in V$ no trecho de $i \in N$ a $j \in N$ pelo número de vagões sendo transportados no trecho. A função objetivo deve ser minimizada. 
As Restrições (3) garantem que o peso total de vagões vazios transportados para atendimento à demanda respeite o limite da folga de capacidade de tração do trem no trecho de $i \in N$ a $j \in N$. As Restrições (4) garantem a conservação de fluxo da quantidade de vagões vazios no nó correspondente no tempo $t=1$, considerando a oferta e/ou demanda, bem como os vagões que foram enviados para outros nós. Nessas restrições, ocorre o momento inicial de planejamento de cada pátio, ou seja, ainda não existem vagões que podem chegar via trem para tais nós.

As Restrições (5) diferem das Restrições (4), pois representam os outros tempos, $t>1$, no vetor espaço-tempo de certo pátio e, assim, consideram também os vagões vazios que chegam de outros pátios em tempos anteriores via trem para o cálculo da conservação de fluxo, o que não ocorre nas Restrições (4).

As Restrições (6) garantem que a quantidade de vagões vazios transportados para atendimento à demanda, somada à quantidade de vagões atuais no trem, no trecho $i \in N$ a $j \in N$, respeite a quantidade máxima de vagões permitida em um trem no trecho.

As Restrições (7) garantem que não haja fluxo de vagões vazios de um pátio em certo tempo para o mesmo pátio no mesmo tempo, $i=j$. As Restrições (8) e (9) definem o domínio das variáveis.

\section{ESTUDO DE CASO E GERAÇÃO DAS INSTÂNCIAS}

Atualmente, a FCA utiliza trens formados exclusivamente de vagões vazios para realizar a distribuição de vagões vazios. 0 modelo proposto, como visto anteriormente, tem por objetivo utilizar a folga de capacidade de tração dos trens carregados que já circulam na ferrovia para realizar a distribuição de vagões vazios visando a redução dos trens exclusivos de vagões vazios. Assim sendo, será analisado se essa estratégia de distribuir vagões vazios utilizando a folga de capacidade dos trens pode reduzir a formação de trens que transportam exclusivamente vagões vazios, que não geram receita para a ferrovia e geram custos com maquinista e diesel.

Para exemplificar a estratégia proposta de utilização da folga na capacidade de tração em trens carregados, considera-se que uma ferrovia só disponha de locomotivas de $4.000 \mathrm{HP}$ e é necessário formar um trem que demanda $6.000 \mathrm{HP}$ de potência para deslocar o peso dos vagões carregados. Assim, deve-se acoplar duas locomotivas que somadas entregam $8.000 \mathrm{HP}$ de potência, gerando, assim, uma folga de tração de 2.000 HP. Dessa forma, poder-se-ia acoplar um conjunto de vagões vazios que demandasse até os $2.000 \mathrm{HP}$ de folga na capacidade de tração na cauda do trem, evitando assim, a formação de um trem exclusivamente de vagões vazios.

Os dados para elaboração das instâncias foram levantados com base no sistema de informação da FCA e com base em entrevistas realizadas com os profissionais da operação da ferrovia, responsáveis pela programação e distribuição de vagões da FCA. A FCA é uma ferrovia que tem seu tráfego, medido em número de trens por dia, baseado na sua grande maioria em contratos de longo prazo para transporte de soja, farelo de soja e produtos siderúrgicos. Por conta disso, o número de trens que circula todos os dias na ferrovia tende a se repetir ao longo dos dias de todos os meses do ano. Assim sendo, foram escolhidos três meses do ano de 2014, julho, agosto e setembro, para elaborar doze instâncias para testar o modelo matemático proposto. Cada uma das doze instâncias testadas representa uma semana de operação da ferrovia FCA com distribuição de vagões vazios. Na Tabela 1 , são apresentadas as doze instâncias que serão testadas. 
Tabela 1: Instâncias para avaliação do modelo matemático

\begin{tabular}{llll}
\hline Instância & Período da Análise & No de Pátios (un) & Horizonte de Tempo (un) \\
\hline 1 & julho/14 & 3 & 7 \\
2 & julho/14 & 5 & 7 \\
3 & julho/14 & 7 & 7 \\
4 & julho/14 & 10 & 7 \\
5 & agosto/14 & 10 & 7 \\
6 & agosto/14 & 12 & 7 \\
7 & agosto/14 & 15 & 7 \\
8 & agosto/14 & 20 & 7 \\
9 & semana 1 - setembro/14 & 30 & 7 \\
10 & semana 2 - setembro/14 & 30 & 7 \\
11 & semana 3 - setembro/14 & 30 & 7 \\
12 & semana 4 - setembro/14 & 30 & 7 \\
\hline
\end{tabular}

As Instâncias 1 a 8 representam casos de teste baseados nos dados extraídos de diversos corredores da FCA nos meses de julho e agosto de 2014. As Instâncias de 9 a 12 representam casos reais, sendo que cada uma representa uma semana do mês de setembro de 2014, de modo a permitir a comparação entre a resolução do modelo matemático proposto e aquela realizada pelo processo manual da FCA. Todas as instâncias consideram um horizonte de planejamento de uma semana, 7 dias.

Por questões de confidencialidade dos dados da FCA, o custo para formação de trens exclusivamente de vazios e o custo de acoplar vagões em trens carregados não podem ser divulgados. Dessa forma, adotou-se o valor informado pela FCA somado a uma constante não divulgada para o cálculo da função objetivo do modelo.

\section{APRESENTAÇÃO E ANÁLISE DOS RESULTADOS}

Foi utilizado o solver CPLEX 12.6 (IBM, 2017) para executar o modelo matemático proposto e foram avaliados os seguintes parâmetros: Tempo de execução, GAP e Função Objetivo. Além desses três parâmetros foi avaliada a quantidade de trens formados exclusivamente com vagões vazios pelo processo manual atualmente realizado pela FCA e na solução encontrada pelo CPLEX. O CPLEX foi executado em um computador com processador Intel i7 com 16 GB de memória RAM. Os resultados obtidos pelo CPLEX podem ser vistos na Tabela 2.

Tabela 2: Resultados do CPLEX

\begin{tabular}{llll}
\hline Instância & Função Objetivo (R\$) & Tempo de Execução (s) & GAP (\%) \\
\hline 1 & $6.350 .000,0$ & 4,5 & 0,0 \\
2 & $7.851 .500,0$ & 5,3 & 0,0 \\
3 & $9.534 .500,0$ & 6,2 & 0,0 \\
4 & $10.193 .250,0$ & 7,4 & 0,0 \\
5 & $8.125 .000,0$ & 7,0 & 0,0 \\
6 & $11.315 .000,0$ & 8,3 & 0,0 \\
7 & $15.326 .000,0$ & 12,7 & 0,0 \\
8 & $22.143 .050,0$ & 18,5 & 0,0 \\
9 & $29.587 .000,0$ & 20,8 & 0,0 \\
10 & $37.550 .000,0$ & 22,1 & 0,0 \\
11 & $36.950 .000,0$ & 20,4 & 0,0 \\
12 & $31.170 .050,0$ & 19,5 & 0,0 \\
\hline
\end{tabular}

Pode-se ver que o CPLEX conseguiu encontrar soluções ótimas para todas as instâncias testadas, mesmo para as instâncias reais, Instâncias 9 a 12. Para a Instância 10, com maior tempo 
de processamento, o CPLEX precisou de um tempo de aproximadamente 22 segundos para obtenção da solução ótima, um tempo muito pequeno de execução para se chegar ao planejamento semanal de distribuição de vagões vazios. Esse tempo indica que o CPLEX pode ser adotado para realizar o replanejamento todo dia caso ocorra algum evento que altere as premissas de planejamento.

Para avaliação da estratégia proposta, conforme apresentado anteriormente, foram analisados os resultados das Instâncias 9 a 12, as quais representam cenários reais da ferrovia. A Tabela 3 apresenta quantos trens exclusivos para distribuir vagões vazios que os distribuidores de vagões da FCA tiveram que formar e circular para fazer a distribuição de vagões vazios visando atender à demanda (processo manual) e a quantidade de trens exclusivos de vagões vazios que o CPLEX utilizou após ter realizado a distribuição de vagões vazios, de forma ótima, utilizando a folga de tração dos trens carregados.

Tabela 3: Comparação das instâncias reais para avaliação da estratégia proposta

\begin{tabular}{ccccc}
\hline \multirow{2}{*}{ Instância } & \multicolumn{2}{c}{ № de Trens de Vagões Vazios } & \multicolumn{2}{c}{ Redução № Trens CPLEX x FCA } \\
\cline { 2 - 5 } & FCA (un) & CPLEX (un) & № de trens (un) & Percentual (\%) \\
\hline 9 & 2 & 2 & 0 & 0,0 \\
10 & 4 & 3 & 1 & $-25,0$ \\
11 & 4 & 1 & 3 & $-75,0$ \\
12 & 3 & 1 & 2 & $-50,0$ \\
\hline
\end{tabular}

Observa-se que na $1^{\text {a }}$ semana de setembro/14, referente à Instância 9, o modelo proposto obteve solução ótima formando a mesma quantidade de trens de vagões vazios formada pelo processo manual da ferrovia. Para as demais semanas do mês de setembro/14, referente às Instâncias 10 a 12, o modelo conseguiu formar menos trens exclusivamente de vagões vazios que aqueles de fato utilizados pela programação da ferrovia no mesmo período. Ou seja, em todos os cenários o CPLEX conseguiu formar menos trens exclusivamente de vagões vazios ou, na pior das hipóteses, a mesma quantidade.

Analisando as quatro semanas do mês de setembro/14, Instâncias 9 a 12, os distribuidores de vagões da FCA formaram 13 trens exclusivamente de vagões vazios para atendimento às demandas dos pátios, enquanto que o CPLEX, rodando o modelo proposto, após ter otimizado toda a folga de tração disponível nos trens em circulação, utilizou um total de 7 trens exclusivos de vazios para atendimento às demandas dos pátios no período em análise, representando uma redução global de aproximadamente $46,2 \%$.

Por formar menos trens exclusivos de vagões vazios, a diminuição desses trens na ferrovia permite liberar segmentos na via para a circulação de mais trens de vagões carregados, que são efetivamente os trens que geram receita para a ferrovia, ou ainda para realizar serviços de manutenção da via.

Vale ressaltar que distribuir vagões por meio de trens formados exclusivamente de vagões vazios gera um custo muito alto para a ferrovia, pois deverá ser alocada uma locomotiva e um maquinista para um trem que não gera receita. Em contrapartida, a estratégia proposta neste artigo, distribui os vagões vazios para atender a demanda com um custo muito inferior, por aproveitar a folga de capacidade dos trens carregados que já circulam na ferrovia.

Assim sendo, o modelo matemático proposto baseado na estratégia de distribuição de vagões vazios utilizando a folga de tração dos trens de vagões carregados proposta neste artigo se mos- 
trou viável e pode trazer resultados significativos para a FCA. Mais ainda, esse modelo utilizando a estratégia pode ser adotado por qualquer outra ferrovia brasileira ou estrangeira.

\section{CONCLUSÔES}

Este artigo apresentou um modelo matemático de Programação Inteira baseado na estratégia de distribuição de vagões vazios que tem por objetivo minimizar o custo total da distribuição de vagões, utilizando ao máximo a folga na capacidade de tração dos trens carregados em circulação na ferrovia.

Testes foram realizados com dados da FCA utilizando o solver CPLEX, o qual conseguiu resolver otimamente todas as instâncias com tempos de execução compatíveis aos horizontes de planejamento propostos. Nas instâncias testadas, baseadas em dados reais da FCA, o CPLEX, rodando o modelo proposto, utilizou menos trens formados exclusivamente de vagões vazios ou, na pior das hipóteses, a mesma quantidade, para distribuir vagões do que os efetivamente criados pelos distribuidores de recursos da ferrovia no período analisado, representando uma redução global de aproximadamente 46,2\%. Por formar menos trens exclusivos de vagões vazios, a diminuição de trens na ferrovia permite liberar segmentos na via para a circulação de mais trens de vagões carregados, que são efetivamente os trens que geram receita para a ferrovia.

Conclui-se, portanto, que o modelo matemático utilizando a estratégia proposta pode ser aplicado como ferramenta de gestão e planejamento da distribuição de vagões em pátios ferroviários para atendimento à demanda de formação de trens em qualquer ferrovia, tanto no Brasil, quanto no exterior.

Sugere-se, como trabalhos futuros, que seja desenvolvida uma heurística para o modelo matemático proposto a fim de facilitar a incorporação do modelo matemático utilizando a estratégia proposta aos sistemas legados das ferrovias.

\section{REFERÊNCIAS}

BEKTAŞ, Tolga; CRAINIC, Teodor Gabriel; MORENCY, Vincent. Improving the performance of rail yards through dynamic reassignments of empty cars. Transportation Research Part C: Emerging Technologies, v. 17, n. 3, p. 259-273, 2009. https://doi.org/10.1016/j.trc.2008.11.003

CONFEDERAÇÃO NACIONAL DO TRANSPORTE (CNT). Anuário CNT do Transporte. Brasília: CNT, 2017.

CORDEAU, Jean-Francois; TOTH, Paolo; VIGO, Daniele. A survey of optimization models for train routing and scheduling. Transportation Science, v. 32, n. 4, p. 380-404, 1998. https://doi.org/10.1287/trsc.32.4.380

COSTA, J. C. Algoritmo de Distribuição e Alocação Vagões em Tempo Real. Dissertação de Mestrado, UNICAMP, 2010.

CRAINIC, Teodor Gabriel; LAPORTE, Gilbert. Planning models for freight transportation. European Journal of Operational Research, v. 97, n. 3, p. 409-438, 1997. https://doi.org/10.1016/S0377-2217(96)00298-6

DEJAX, Pierre J.; CRAINIC, Teodor Gabriel. Survey paper-a review of empty flows and fleet management models in freight transportation. Transportation Science, v. 21, n. 4, p. 227-248, 1987. https://doi.org/10.1287/trsc.21.4.227

FUKASAWA, R. Resolução de problemas de logística ferroviária utilizando programação inteira - Dissertação de Mestrado, PUC-RJ, 2002.

HAGHANI, Ali E. Formulation and solution of a combined train routing and makeup, and empty car distribution model. Transportation Research Part B: Methodological, v. 23, n. 6, p. 433-452, 1989. https://doi.org/10.1016/0191-2615(89)90043-X

HOLMBERG, Kaj; JOBORN, Martin; LUNDGREN, Jan T. Improved empty freight car distribution. Transportation Science, v. 32, n. 2, p. 163-173, 1998. https://doi.org/10.1287/trsc.32.2.163

IBM. CPLEX Optimization Studio. Disponivel em: <https://www.ibm.com/br-pt/>. Acesso em: 6 abr. 2017.

JOBORN, Martin et al. Economies of scale in empty freight car distribution in scheduled railways. Transportation Science, v. 38, n. 2, p. 121-134, 2004. https://doi.org/10.1287/trsc.1030.0061

MELO, M. C. V.; BARROS NETO, J. F.; NOBRE Jr, E.F. Problema de Planejamento da Alocação de Vagões de Carga: uma Abordagem via Modelagem Matemática. Transporte em Transformação XIII, 2008.

MISRA, S. C. Linear programming of empty wagon disposition. Rail International, v. 3, n. 3, 1972.

PHILIP, C.E. Freight car utilization and railroad reliability: the application of an inventory model to the railroad empty car distribution process. Technical Report MIT MIT-CTS-78-2, Center for Transportation Studies, Cambridge, MA, 1978. 
POWELL, Warren B.; BOUZAIENE-AYARI, Belgacem; SIMAO, Hugo P. Dynamic models for freight transportation. Handbooks in operations research and management science, v. 14, p. 285-365, 2007.

POWELL, Warren B.; CARVALHO, Tassio A. Real-time optimization of containers and flatcars for intermodal operations. Transportation Science, v. 32, n. 2, p. 110-126, 1998. https://doi.org/10.1287/trsc.32.2.110

POWELL, W.B., TOPALOGLU, H. Fleet management. In: Wallace, S., Ziemba, W. (Eds.), Applications of Stochastic Programming Math Programming Society-Series on Optimization. SIAM, Philadelphia, 2005.

SHERALI, Hanif D.; SUHARKO, Arief B. A tactical decision support system for empty railcar management. Transportation Science, v. 32, n. 4, p. 306-329, 1998. https://doi.org/10.1287/trsc.32.4.306

SPIECKERMANN, Sven; VOß, Stefan. A case study in empty railcar distribution. European Journal of Operational Research, v. 87, n. 3, p. 586-598, 1995. https://doi.org/10.1016/0377-2217(95)00232-4

WHITE, William W..; BOMBERAULT, Abel M. A network algorithm for empty freight car allocation. IBM Systems Journal, v. 8, n. 2, p. 147-169, 1969. 